\title{
Analysis of Phased Aperture Array Geometries for Low Frequency Radio Astronomy`
}

\author{
N. Razavi-Ghods ${ }^{1}$, E. de Lera Acedo ${ }^{1}$, A. El-Makadema ${ }^{2}$, P. Alexander ${ }^{1}$, A. Brown ${ }^{2}$ \\ 1 Cavendish Laboratory, University of Cambridge, CB3 0HE, Cambridge UK \\ 2 School of Electrical and Electronic Engineering, The University of Manchester, Manchester, M60 1QD UK
}

\begin{abstract}
The proposed SKA telescope will rely heavily on the use of aperture phased arrays in the sub $1 \mathrm{GHz}$ frequency band. Currently two different arrays (from nominally $70 \mathrm{MHz}$ to $450 \mathrm{MHz}$ and from $300 \mathrm{MHz}$ to $1 \mathrm{GHz}$ ) are being studied for inclusion within the overall SKA configuration. In this paper we focus on analysing the real sky contribution to system temperature for various AA-lo antenna configurations composed of 10,000 elements. We evaluate six geometries, with four minimum inter-element separations of $0.5 \lambda, 0.8 \lambda, 1 \lambda$, and $2 \lambda$. We assume uniform excitation of antennas as well as Taylor and Dolph-Chebyshev weighting with a side-lobe level of $35 \mathrm{~dB}$. Although the simulations are tuned to observations at $100 \mathrm{MHz}$, the results can be temperature scaled to the appropriate frequency band. Our analysis of array temperature is carried out by assuming observations of three cold regions above and below the Galactic plane. The results show comparisons between regular and random array geometries.
\end{abstract}

\section{Introduction}

The SKA Design Study (SKADS) is an international effort to explore and develop technologies which will enable the construction of the next generation radio astronomy telescope with over a million square meters of collecting area, the size of which will provide two orders of magnitude more sensitivity than current radio instruments (www. skatelescope.org). The proposed frequency range of the SKA is $0.07-10 \mathrm{GHz}$ and this will be made possible using multiple collector technologies, consisting of dishes at high frequencies and aperture arrays for low frequency ranges, where the key science objective is detecting red-shifted hydrogen emission at around $1 \mathrm{GHz}$ and below. For hydrogen surveys, it is critical to achieve high sensitivity, bandwidth, and field of view (FoV) leading to high survey speeds (Abdalla et al. 2009).

The types of aperture arrays studied so far have consisted mainly of densely packed antenna arrays in the higher frequency regime up to of the order $1 \mathrm{GHz}$ as demonstrated by a number of systems, namely the early THEA (Smolders \& Kant 2000) and the current EMBRACE (van Ardenne et al. 2004) and 2-PAD (http: //2-pad.physics . ox . ac . uk) programmes. Below $500 \mathrm{MHz}$, however, there are many issues which become apparent mainly because of the sheer size of the antennas and the budgetary limitations of the SKA given the proposed specifications (Schilizzi et al. 2007). In particular, the number of antennas used in the array and as well as the cost of materials. Due to the high sky temperature, the effective area of the overall array is critical in this design. This has led to thinned or sparse arrays being adopted at low frequency bands to minimize the number of active antennas used

* This work was supported by the European Commission Framework Program 6, Project SKADS, Square Kilometre Array Design Studies (SKADS), contract no 011938. in order to provide a given sensitivity (Bolton et al. 2008). As an example, the LOFAR system operates from $30-240 \mathrm{MHz}$, in two concurrent bands using different antenna array geometries in each (www . lofar .org). It is desirable for the SKA low frequency array (AA-lo) to operate using a single collector technology between (nominally) $70 \mathrm{MHz}$ and $450 \mathrm{MHz}$ and thus a single antenna array geometry. The question of the array geometry is therefore a critical one, since this, along with the antenna weighting scheme, defines the sidelobe level and profile, all of which contribute to the antenna temperature and sensitivity of the array.

In order to evaluate accurately these arrays and form an intuitive goodness criterion, the contribution from the real sky must be taken into account. For example, a sidelobe $30 \mathrm{~dB}$ below the main peak can have significant impact on the sensitivity if that sidelobe sits on a region of sky with a mean temperature 1000 times the receiver temperature. This is important at low frequencies where the Rayleigh-Jeans temperature of the Galaxy can reach over $2000 \mathrm{~K}$ at $500 \mathrm{MHz}$. Furthermore, some so-called sparse arrays proposed for the SKA result in grating lobes which have major consequences for such a radio astronomy instrument. Fig. 1 shows the far field response of an undersampled $(0.8 \lambda$ spacing $)$ triangular lattice array, where the grating lobe $(<10 \mathrm{~dB})$ is pointing towards the galactic plane. This type of array therefore presents a number of calibration and signal processing challenges.

In this paper we evaluate a number of different array geometries, namely, regular, triangular, circular, thinned $(25 \%)$, sparse $(25 \%)$ and fully randomized. For each array, the resulting beam function is computed and convolved with the frequency corrected sky model for an observation period of approximately four and a half hours. The result of this calculation is the array temperature which is the primary figure of merit used in this paper. Section 2 describes various phased array ge- 

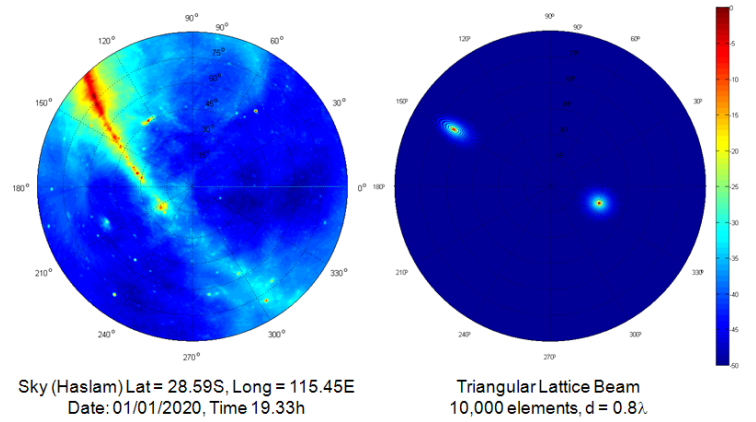

Fig. 1: Grating lobe of a triangular lattice array pointing towards the galactic plane at $1000 \mathrm{~K}(500 \mathrm{MHz}$.)

ometries and weighting schemes. Section 3 briefly describes the AA-lo antenna. In section 4, we describe the simulations carried out, and conclude the paper in sections 5 .

\section{Array geometries and weighting}

Regular sampled aperture phased arrays have been studied extensively so far and their behavior is well documented in Hansen (1998) and Mailloux (2005). In particular it is known that for a regular lattice grating lobes will result when the following equality is exceeded

$$
\frac{d}{\lambda}=\frac{1}{1+\sin (\theta)}
$$

where $d$ is the inter-element spacing and $\theta$ is the scan angle. Given that the proposed scan range for the low frequency SKA is \pm 45 degrees from zenith (Bolton et al. 2008), this implies a minimum element separation of $\sim 0.6 \lambda$. Eq. (1) is only valid when the array has regular inter-element spacing. For the case of random arrays, the power present in the grating lobes of the regular array are now re-distributed into many smaller and "random" sidelobes. This has been a useful feature of sparse random arrays as described in Braun \& W. van Cappellen et al. (2006) and is the reason that they are of interest to the SKA community. Furthermore, if each SKA station has a randomized design, the cross-correlation between stations in an interferometer would suppress the sidelobes in the cross-power beam.

Irregular arrays, however, are not an ideal solution. Increasing the minimum inter-element spacing produces sidelobes which reduce the array gain by a significant factor. Fully randomizing the antenna positions may be an optimal solution since we would expect to randomize both the overall array sidelobes and also possible degradations due to mutual coupling between array elements. However, a more appropriate option may be to specifically tailor the antenna locations to a specific profile - that is a deterministic approach to locating antennas in an under sampled array. The six geometries examined in this paper are shown in Fig. 2

Element weighting is also one of the primary design parameters of such arrays. It is well known that various techniques can be used to control the sidelobe profiles of fully sampled phased arrays from simple Taylor weighting (Taylor

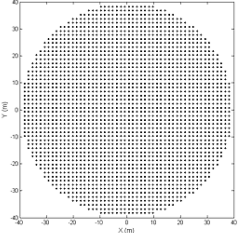

(a)

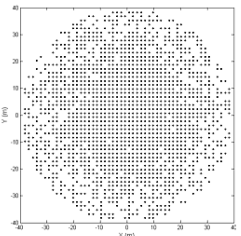

(d)

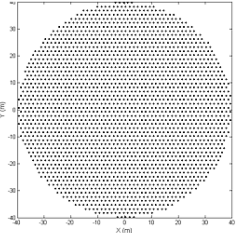

(b)

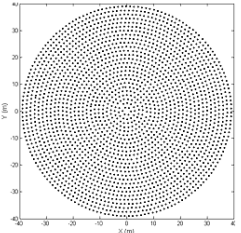

(e)

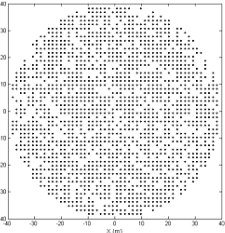

(c)

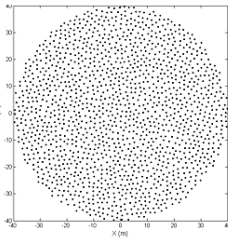

Fig. 2: Planar phased array geometries: (a) regular, (b) triangular, (c) sparse random (25\%), (d) Thinned (25\%), (e) Circular, (f) true random.

1955) to adaptive nulling (Fenn 2007). The Fourier series design method (Oppenheim \& Schafer 1989) which is based on the inverse discrete-space Fourier transform of the array factor is also useful for obtaining the element weights corresponding to a desired response but suited to very large (ideally infinite) arrays. In this paper we restrict our investigation by considering only Dolph-Chebyshev (Dolph 1946) and Taylor weighting as applied to the different array geometries studied. DolphChebyshev weighting achieves a constant sidelobe level whilst Taylor weighting gives a more practical distribution of sidelobes with a sinc type reduction. Both weighting schemes have their inherent advantages.

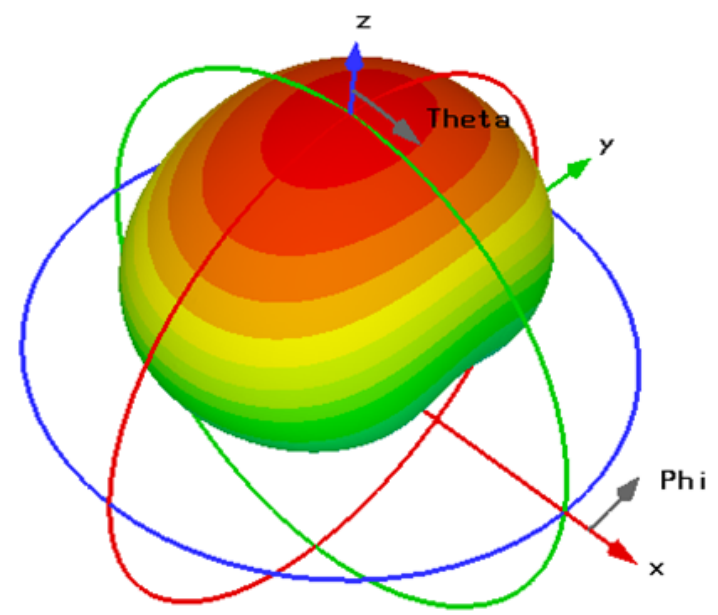

Fig. 3: Bow-Tie antenna radiation pattern (CST package).

\section{AA-lo Antenna}

The design of a single aperture array antenna element for the low frequency band of the SKA requires good impedance and 
noise stability across a $\sim 6.5: 1$ frequency band as well as the appropriate forward gain in an array environment. Also, broad beam-widths of at least \pm 45 degrees are needed for the embedded element patterns in order to achieve the desired scan range. This is a major challenge and the focus of ongoing research in the SKA community. One such candidate is the Bow-Tie antenna presented in de Lera Acedo et al. (2009). This antenna's radiation pattern is used in the simulations presented in this paper (Fig. 3).

\section{Numerical simulations and results}

The array temperature was found by multiplying the normalized array beam (computed numerically using NFFT) with the sky brightness function at $100 \mathrm{MHz}$ and integrating over the whole sky (Cortes Medellin 2007). In our simulations we used data from the $408 \mathrm{MHz}$ survey (conducted by Haslam et al. 1981). We evaluated the array temperature over three cold regions as shown in Fig. 4, which is the SKA aperture array observable sky if the telescope was located in one of two candidate sites.

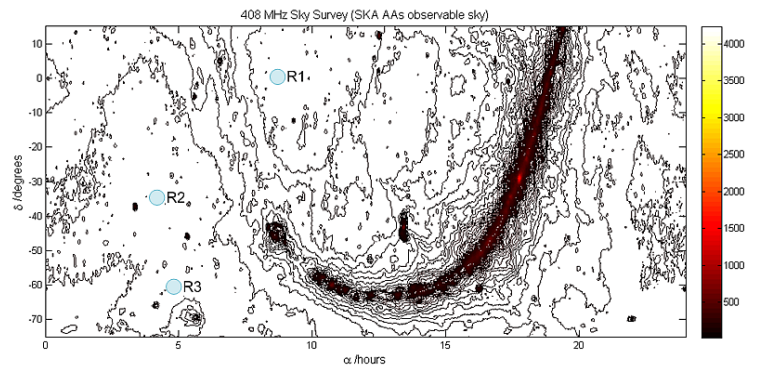

Fig. 4: Observable sky (at $408 \mathrm{MHz}$ ) for the SKA aperture arrays.

The array temperature for regions 1 and 2 are shown in Fig. 5 and Fig. 6, respectively. Region 3 data is omitted here as it is similar to region 2 .

Fig. 6 shows results which might be typically expected, where as the array inter-element spacing in cartesian or radial directions increases, the array temperature also increases. Perhaps where the smoothest transition happens is for a random grid. Grating lobes do play a role here but are not as significant as for region 1.

Fig. 5, shows a very different scenario. Here the grating lobes fall right on top of the galactic plane resulting in a major spike in array temperature. These results also show that the $1 \lambda$ arrays are worst than $2 \lambda$ arrays. The reason for this is that although the positions of the grating lobes correspond, their beam-width do not, leading to a much wider beam pointed to a hot region for the $1 \lambda$ case which results in a higher temperature spike.

In this case, most of the arrays which have an underlying regular structure suffer with the exception of circular and random arrays. For the circular array it is expected that grating lobes would be present in the radial direction and would eventually result in similar temperature spikes.
The use of weighting schemes such as Taylor or DolphChebyshev do have their advantages if the primary figure of merit is the array temperature and not effective area. Both schemes result in a reduction of the primary sidelobes which reduce the overall sky contribution to system temperature. Taylor weighting achieves a modest improvement over DolphChebyshev, however for cases such as region 1 it provides a far better improvement in array temperature. As expected, both weighting schemes have the effect of reducing the gain of the array and thus the effective area leading to a lower overall sensitivity.
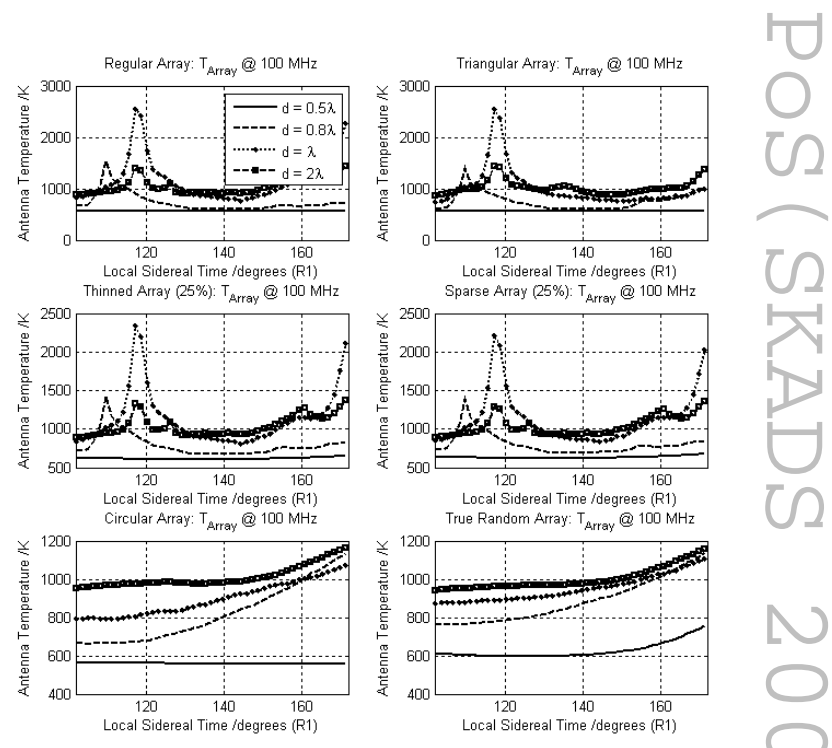

Fig. 5: Array Temperature for Region 1.
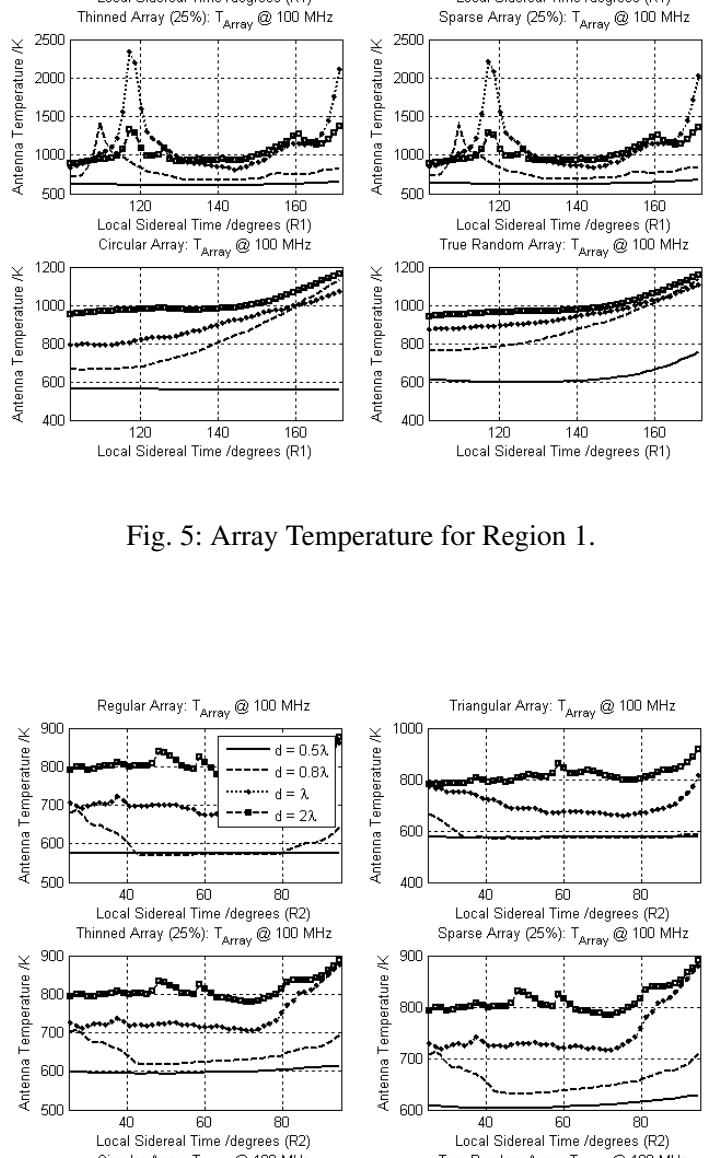

Circular Array: T
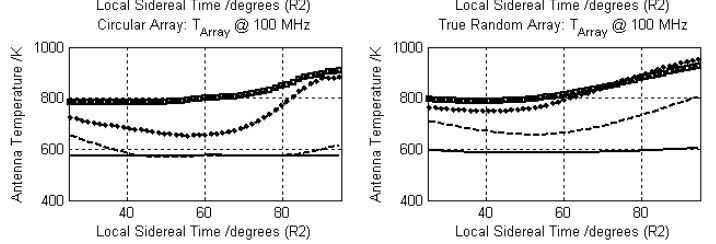

Fig. 6: Array Temperature for Region 2. 


\section{Conclusions}

This paper describes simulations carried out to evaluate array temperature for six different AA-lo geometries with four interelement separations and two weighting schemes.

Although the results are dependant on the region(s) being scanned, it is apparent that grating lobes do have a significant impact on array temperature. Random arrays show a much smother temperature profile as the array spacing increases and they do not suffer from grating lobes. However further work needs to be carried out to assess more accurately how the far out and near in sidelobes impact the results, particularly for random array configurations.

Acknowledgements. We are grateful to our SKA colleagues for several discussions around this topic.

\section{References}

Abdalla, F., C. Blake \& S. Rawlings, "Forecasts for Dark Energy Measurements with Future HI Surveys," MNRAS, arXiv:0905.4311v1 [astro-ph.CO]

van Ardenne, A., P.N. Wilkinson, P.D. Patel \& J.G. Bij De Vaate, "Electronic Multi-beam Radio Astronomy Concept: Embrace a Demonstrator for the European SKA Program," Experimental Astronomy, Vol. 17, No. 1-3, pp. 65-77, June 2004.

Bolton R.C., et al., "SKADS Benchmark Scenario Design and Costing 2 (The SKA Phase 2 AA Scenario)," Oct. 2008 http: //www . skads-eu .org/PDF/SKADS_D\&C_ second_document_05112008_final.pdf

Braun, R. \& W. van Cappellen, SKA Memo 87: "aperture arrays for the SKA: dense or sparse?" Available online: http: //wWw . skatelescope.org/PDF/memos/memo_87.pdf

Cortes Medellin, G., SKA Memo 95: "Antenna Noise Temperature Calculation," Available online: http://www . skatelescope.org/PDF/memos/Memo_95.pdf

Dolph, C.L., "A Current Distribution for Broadside Arrays Which Optimizes the Relationship Between Beam Width and Side-Lobe Level," Proc. IRE Waves Electrons, 34, 335, June 1946.

Fenn, A.J., "Adaptive Antennas and Phased Arrays for Radar and Communications," New York: Artech House, 2007.

Hansen, R.C., "Phased Array Antennas," New York: John Wiley \& Sons, 1998.

Haslam, C.G.T., U. Klein, C.J. Salter et al., A 408 MHz all-sky continuum survey. I Observations at southern declinations and for the North Polar region, Astronomy \& Astrophysics, vol. 100, no. 2, pp. 209-219, July 1981.

de Lera Acedo, E., N. Razavi-Ghods, E. Garcia, P. J. Duffett-Smith \& Paul Alexander, System Noise Analysis of an Ultra Wide Band Aperture Array Element for Low Frequency Radio Astronomy, in Proceedings of the 6th IASETD International Conference Antennas, Radar, and Wave Propoagation (ARP 2009), Banff, Alberta, Canada, July 6-8, 2009.

Mailloux, R.J., Phased Array Antenna Handbook, 2 Ed. New York: Artech House, 2005.
Oppenheim, R.W., \& Schafer, A.V., "Discrete Time Signal Processing," Prentice Hall, Upper Saddle River, NJ, 1989.

Schilizzi, R.T., SKA Memo 100: "Preliminary Specifications for the Square Kilometre Array," Dec. 2007 http://www.skatelescope.org/PDF/memos/ 100_Memo_Schilizzi.pdf

Smolders \& G.W. Kant, A.B., Thousand Element Array (THEA), IEEE Antennas and Propagation Society International Symposium 2000, pp 162 - 165 vol.1, July 2000.

Taylor, T.T., Design of line source antenna for narrow beamwidth and low sidelobes, IRE Trans. Antennas Propagat., vol. AP-4, pp. 16-28, January 1955. 